\title{
Isolated Hippocampal Infarct and Dementia - A Case Report and Literature Review
}

\author{
Vimal Mannali ${ }^{* *}$ and Sujoy Mukherjee ${ }^{2}$ \\ West London Mental Health Trust, Cognitive Impairment and Dementia Service (CIDS) Ealing West, Greenford, Greater London, United Kingdom \\ *Correspondence to: Vimal Mannali, Speciality Registrar ST6, West London Mental Health Trust, Cognitive Impairment and Dementia Service- CIDS Ealing West, \\ Elm Lodge, 4a Marley Close, Greenford, Greater London,UB6 9UG, United Kingdom; Email: vimal.mannali@wlmht.nhs.uk
}

Received: December 09, 2017 Accepted: December 11, 2017; Published: December 12, 2017;

\begin{abstract}
Ischaemic stroke in the Hippocampal regions have been characteristically described as resembling clinical picture of Transient Global Amnesia (TGA). Prolonged and persistent cognitive deficits leading to a diagnosis of Dementia, within the context of Hippocampal lesions, have been less widely reported. Routine cognitive screening without a careful exploration of evolution of deficit symptoms might miss the crucial narrative suggestive of a Vascular Dementia of relatively acute onset. Characteristic magnetic resonance imaging finding of localised lesion(s) in the Hippocampus will lend significant diagnostic clarity and aid in formulating an effective care management. Our case report describes the identification of a unilateral isolated infarct in the Left Hippocampus with non-fluctuant cognitive deficits for over 6 months, leading to a diagnosis of Vascular Dementia, and a review of the current literature.
\end{abstract}

Keywords: Dementia, Hippocampus, Infarction, Stroke, Amnesia, Neuroimaging

\section{Background}

Amnesic stroke has been linked to isolated lesions in medial temporal lobe, thalamus, basal forebrain, retro-splenial region, and subcortical regions [1]. Lesions in the medial temporal lobe have been clearly linked to severe amnesia [2]. Several case reports mention that lesions affecting the posterior cerebral artery may cause confusion [3]. Despite diagnostic advances with neuroimaging, unilateral ischaemic lesions within the Hippocampal region continue to be rare.

Case reports mostly mention occurrence of Transient Global Amnesia (TGA) within the context of a Hippocampal Stroke [2,4,5]. Anterograde amnesia found in TGA, with relatively intact immediate recall and retrograde memory, rarely persist beyond 24 hours. TGA with identified unilateral lesions were found to present with confusion, object specific hallucinations, intense mood states changes, and impulsivity, with or without depressive behaviour [5]. A Case report noted persistent memory deficits lasting up to 5 months following Hippocampal Infarction [6]. Another report illustrated amnesia irreversibility in bilateral Hippocampal Infarction [7]. Post mortem studies have linked permanent Amnesia to Bitemporal stroke [2,4].

Research into identification and characterisation of stroke lesions or patterns affecting Hippocampus has tried to shed light into understanding patterns of cognitive deficits within verbal and nonverbal episodic long-term memory loss and spatial disorientation [4]. Delayed focal changes in the lateral aspect of Hippocampus have been identified on magnetic resonance imaging, in cases of Transient Global Amnesia (TGA) [6]. Lesional studies have described that these memory deficits are in line with commonly seen temporal lobe pathologies [7]. Kumral et al. [8], have described 5 topographic variants of Hippocampal infarcts and described Hippocampal dementia in 2 patients with severe immediate, delayed verbal and visual memory deficits, dysexecutive syndrome, deficits in responding to feedback and error correction, with dull and aimless appearance lasting for several months.

Despite widened neuroimaging and neuropsychological research, there appear to be limited awareness among front line clinicians about Hippocampal lesions in suspected cases of Dementia. A need for further understanding of mixed vascular dementia and Alzheimer's disease within localised hippocampal and medial temporal lesions have been highlighted by different studies [9].

We aim to add to the increasing published evidence with a strategic Hippocampal Infarct presenting with persistent cognitive deficits, leading to a diagnosis of Vascular Dementia.

\section{Case report}

A 70yr old British-Indian male with no previous contact with secondary mental health services, presented with a 4-month history of impaired memory. Characteristic initial identified symptom was being unable to manage his finances, along with occasions of forgetting his food and medication. Prior to the development cognitive symptoms, he was treated for an accidental finding of Hyponatraemia during routine monitoring investigations.

Collateral history from his family described a rapid onset of cognitive deficits and characteristic stable symptoms without any progression for over 4 months. Reported day-to-day and within-day fluctuations present in attention and concentration. Family had noted significant difficulty with self-managing medication and had taken an 
accidental overdose in this period. Reportedly was going to the toilet at least 15 times per day because he would forget having used the toilet. Behavioural and psychological symptoms included pervasive low mood, anxiety symptoms, apathy and pacing around the house at night. He had been using a walking stick to mobilise outdoor due to new onset of dizziness. In terms of sensory impairment, he has required to use hearing aids in this 4-month period. His speech has been relatively preserved and he has been able to make himself easily understood to others when conversing in his native language Punjabi. However he would engage in repetitive speech. Limited variability was seen in his pre-morbid daily routine and social interactions were preserved though he struggled with reduced levels of motivation and energy. Appeared to have lost interest in news and current affairs, which he used to keenly follow in the past.

He had had his schooling in Punjabi language (grew up in India) and understands basic English. In the United Kingdom he had worked as a baker and a metal factory worker, before being made redundant at the age of 62 .

Significant medical issues include Type 2 Diabetes Mellitus, Essential hypertension, Hypercholesterolaemia, Chronic Kidney Disease stage 3, Tubular adenoma, a history of low serum Vitamin B12 and Asthma. Regular prescribed medication include Clenil Modulite inhaler, Omeprazole, Nifedipine, Repaglinide, Pravastatin, Bisoprolol, Aspirin, Metformin and Losartan. No history of any harmful use of alcohol or nicotine. He denied any historic or current use of illicit substances.

A Mini Mental State Examination (MMSE) done at the time of referral from Primary Care (General Practice) had showed a score of 21/30 indicating a mild level of cognitive impairment. The Rowland Universal Dementia Assessment Scale (RUDAS) revealed a score of 27/30, having lost 1 point on Visuo-constructional Drawing and 2 points on Judgement. This did not identify any significant cognitive deficit. The Neuropsychiatric Inventory (NPI) showed a total score of 27/120, Carer Distress at 13/50, Depression at 3 (Carer Distress of 2), Anxiety at 4 (Carer Distress of 3), Apathy at 8 (Carer Distress of 4), Aberrant motor behaviour at 12 (Carer Distress of 4), Neurovegetative Changes at 9/24 (Carer Distress of 4/10), Pacing around the house at night at 9 (with a Carer Distress of 4). On Bristol Activities of Daily Living Scale (BADLS), he scored 14/60 indicating that he had lost about $23 \%$ of his independent functioning. And on Clinical Global Impression (CGI) he was noted as 3 (mildly ill).

The routine Dementia Screen blood test results showed relatively poor diabetic control and a low serum Ferritin but was otherwise within range. Electro-cardiogram (ECG) showed a sinus rhythm with premature ventricular complexes.

As per standard investigation protocol in the Secondary Care (Cognitive Impairment and Dementia Service), an MRI Brain was requested and the scan report (from axial T2, coronal FLAIR, sagittal $\mathrm{T} 1$ and diffusion weighted images) showed that the ventricles were of normal size and configuration; cranio-cervical junction was normal; there was a generalised volume loss with no particular focal predilection; noted a mature gliotic damage affecting the left hippocampal head and para-hippocampal gyrus which appeared more in keeping with an infarct than a primary neurodegenerative process. There were no other intra-axial areas of abnormal signal intensity, or abnormal mass lesions seen within the brain, brain stem or cerebellum. Conclusion of a Medial Left Temporal infarct with a generalised volume loss was arrived at (figures 1, 2 and 3).

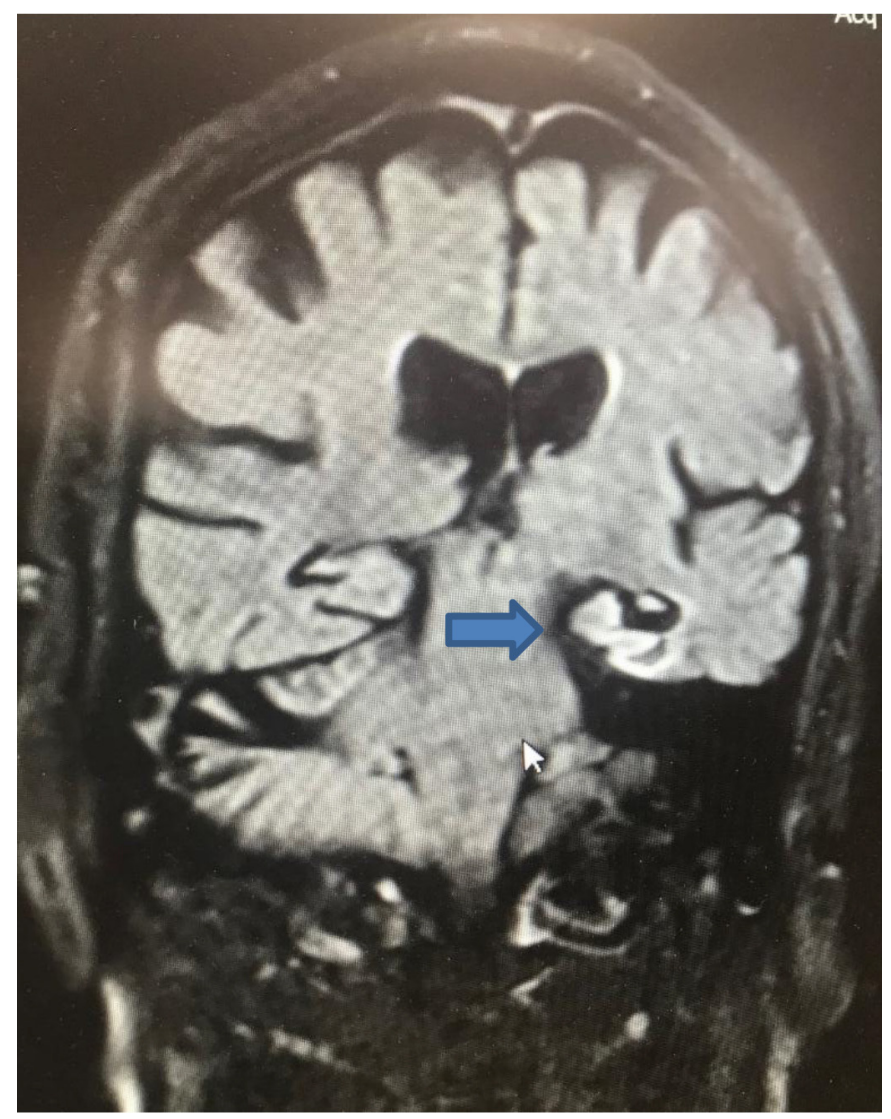

Figure 1. MRI Brain (diffusion-weighted image DWI) coronal view showing strategic infarct on the left Hippocampus (blue arrow)

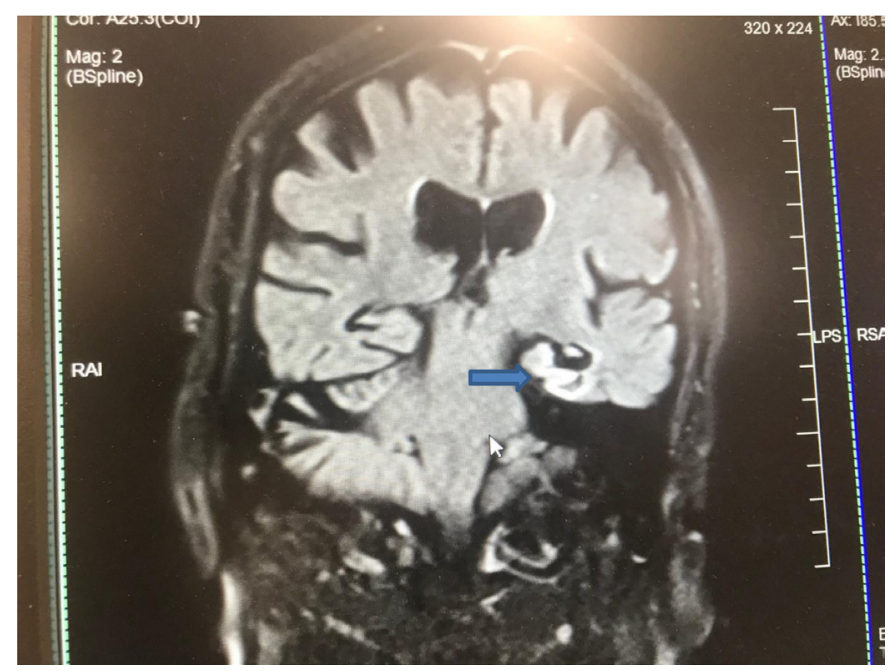

Figure 2. MRI Brain (diffusion-weighted image DWI) coronal view showing strategic infarct on the left Hippocampus (blue arrow) 


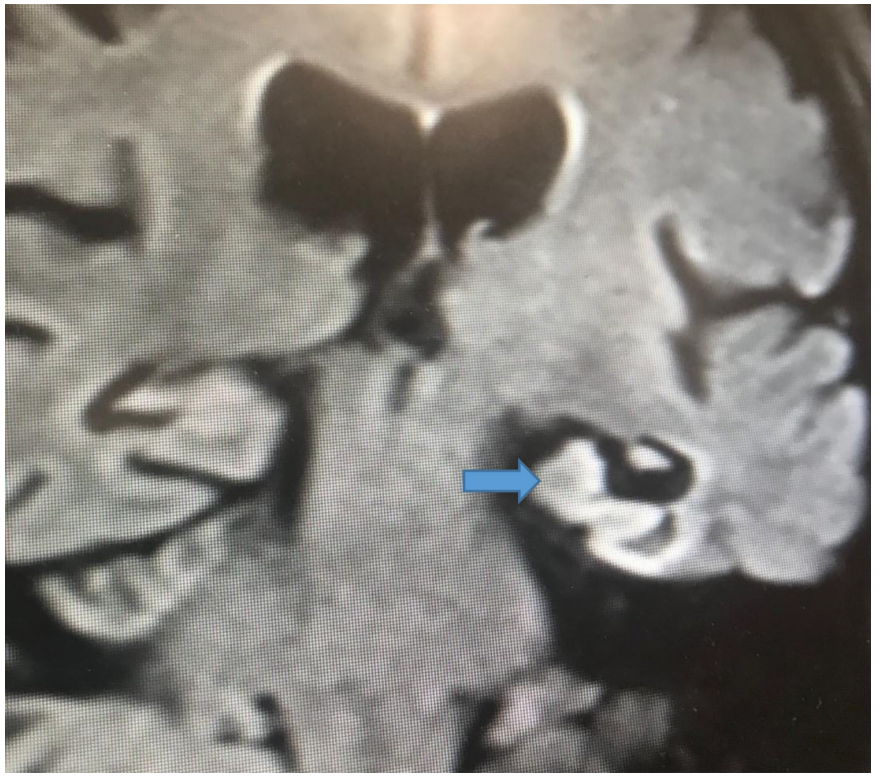

Figure 3. Magnified image of MRI Brain (diffusion-weighted image DWI) coronal view showing strategic infarct on the left Hippocampus (blue arrow)

\section{Discussion}

At initial assessment 4 months following the onset of cognitive symptoms, there was a strong suspicion that this gentleman was suffering from persistent and stable cognitive deficits leaning towards a diagnosis of Dementia. Identification of the strategic infarct in the Left hippocampal region on magnetic resonance imaging was critical to arriving at the diagnosis. However, as the duration of his problem was less than 6 months, it was decided to carefully monitor for any changes to the non-progressive cognitive deficits.

He was not found to be suitable for treatment using Acetyl Cholinesterase Inhibitor given the absence of any clear features suggestive of a concurrent Alzheimer's disease. Was advised to closely monitor his vascular risk factors and highlighted the need for early identification and management of any Delirium, should it occur in the future. Given his depressive symptoms, a trial of antidepressant medication had been suggested. At subsequent follow up (more than six months from initial symptom development), he was found to present with consistent cognitive symptoms and level of functional difficulty.

Our case was unusual having presented with an unresolved anterograde amnesia initially resembling a Transient Global Amnesia (TGA) but the identification of the strategic Hippocampal infarct in a background of significant vascular risk factors, and persistent cognitive symptoms beyond six months, led to a diagnosis of Vascular Dementia. The duration of symptoms at the time of assessment did not allow a formal diagnosis of Dementia but on careful follow up there was persistent and stable cognitive deficits which helped gain diagnostic clarity.

The Sydney Stroke Study [10] showed that patients with Stroke or Transient Ischaemic Attacks (TIA), and patients with post-stroke Mild Cognitive Impairment (Vascular) or mild Vascular Dementia do not have hippocampal atrophy, but they proposed the need for a longitudinal study to determine if hippocampal atrophy is a late development in Vascular Dementia. Focal Cerebral Ischaemia has been widely studied within spatial and temporal evolution of cognitive symptoms. Hippocampal infarctions, both uni and bilateral, are suggested as a direct causation for memory dysfunction and having a potential to lead to irreversible amnestic symptoms $[11,12]$.

Previous studies have identified the difficulty with recognition of small ischaemic strokes in hippocampal regions and subsequent under-diagnosis without adequate follow up of TGA-type episodes, with sole reliance on CT Head scan for neuroimaging [12]. Newer MRI techniques have helped lesional description within stroke patterns affecting the hippocampus [2]. In TGA cases, diffusion-weighted images have revealed small punctuate lesions in the hippocampus [13].

It has been studied that different patterns of hippocampal infarcts result from the variation in arterial supply of hippocampus or suggest location of an embolism [5]. Cardio-embolism and vertebra-basilar artery disease were found as the predominant cerebrovascular mechanisms leading to a Hippocampal infarction [5]. A study found that the CA1 region within the Hippocampal area is most vulnerable to ischemia resulting in TGA, as it lies near the hippocampal blood supply that branches out from the posterior cerebral artery [6]. Another study suggested that their most important finding was absence of cases with isolated infarct of the hippocampus, in contrast to conditions like Herpes simplex encephalitis, Para-neoplastic limbic encephalitis, and primary brain tumours which may predominately or exclusively involve one or both Hippocampi [7]. Case reports have also found other causes for Hippocampal stroke such as glyphosate overdose [14] and induced by Cocaine [15] intoxication.

Our case highlights the need for a careful exploration of historical narrative in evolution of cognitive deficits, the utility of newer MRI techniques in identification of localised Hippocampal lesions and the need for awareness of Medial Temporal Lobe pathologies in secondary and tertiary care settings. Additional neuropsychological examination could help delineating functional deficits and descriptively aid a holistic understanding of the case presentation.

\section{Acknowledgement}

The authors wish to thank the patient and his family for providing consent to share information relevant to this case report and for permission to use the images from his MRI Brain scan.

\section{Competing interest}

The authors declare that they have no competing interests.

\section{Funding information}

The authors declare that they have not received any form of funding for collection of the case information or writing of the case report, and there is no sponsor involved in the decision to submit the paper for publication. 


\section{References}

1. Szabo K, Förster A, Jäger T, Kern R, Griebe M, et al. (2009) Hippocampal Lesion Patterns in Acute Posterior Cerebral Artery Stroke Clinical and MRI Findings. Stroke 40: 2042-2045. [crossref]

2. Szabo K (2014) Hippocampal stroke. Front Neurol Neurosci 34: 150-156. [crossref]

3. Shih HT, Huang WS, Liu CH, Tsai TC, Lu CT, et al. (2007) Confusion or delirium in patients with posterior cerebral arterial infarction. Acta Neurol Taiwan 16: 136142. [crossref]

4. Sander K, Sander D (2005) New insights into transient global amnesia: recent imaging and clinical findings. Lancet Neurol 4: 437-444. [crossref]

5. Li J, Hu WL (2013) Bilateral hippocampal abnormalities in magnetic resonance imaging in transient global amnesia. Am J Emerg Med 31: 755.e1-755.e3. [crossref]

6. Sedlaczek O, Hirsch JG, Grips E, Peters CN, Gass A, et al. (2004) Detection of delayed focal MR changes in the lateral hippocampus in transient global amnesia. Neurology 62: 2165-2170. [crossref]

7. Takahashi S, Higano N, Kurihara S, Mugikura K, Sakamoto H, et al. (1997) Correlation of lesions in the hippocampal region noted on MR images with clinical features. Eur Radiol 7: 281-286. [crossref]

8. Kumral E, Deveci EE, Erdogan C, Enüstün C (2015) Isolated hippocampal infarcts: Vascular and neuropsychological findings. J Neurol Sci 356: 83-89. [crossref]
9. Del Ser T, Hachinski V, Merskey H, Munoz DG (2005) Alzheimer's disease with and without cerebral infarcts. J Neurol Sci 231: 3-11. [crossref]

10. Sachdev PS, Chen X, Joscelyne A, Wen W, Altendorf A, et al. (2007) Hippocampa size and dementia in stroke patients: the Sydney stroke study. J Neurol Sci 260: 71-77. [crossref]

11. Borroni B, Agosti C, Brambilla C, Vergani V, Cottini E, et al. (2004) Is transient global amnesia a risk factor for amnestic mild cognitive impairment? J Neurol 251: 1125-1127. [crossref]

12. Marinkovic I, Lyytinen J, Valanne L, Niinikuru R, Pekkonen E (2012) Bilateral hippocampal infarction as etiology of sudden and prolonged memory loss. Case Rep Neurol 4: 207-211. [crossref]

13. Förster A, Al-Zghloul M, Wenz H, Böhme J, Groden C, et al. (2017) Isolated punctuate hippocampal infarction and transient global amnesia are indistinguishable by means of MRI. Int J Stroke 12: 292-296. [crossref]

14. Nishiyori Y, Nishida M, Shioda K, Suda S, Kato S (2014) Unilateral hippocampal infarction associated with an attempted suicide: a case report. J Med Case Rep 8: 219. [crossref]

15. Morales Vidal SG, Hornik A, Morgan C (2012) Cocaine induced hippocampi infarction. BMJ Case Rep 2012. [crossref]

Citation:

Vimal Mannali, Sujoy Mukherjee (2017) Isolated Hippocampal Infarct and Dementia - A Case Report and Literature Review. Ageing Sci Ment Health Stud Volume 1(2): 1-4 